\title{
Quantitative analysis of the relationship between the LLNA:DAE method results and the LLNA EC3 values highlights the connection between the elicitation and induction phases during skin sensitization
}

\author{
Kunihiko Yamashita ${ }^{1,3}$, Shinsuke Shinoda ${ }^{2}$, Saori Hagiwara ${ }^{2}$ and Hiroshi Itagaki ${ }^{3}$ \\ ${ }^{1}$ Corporate Research Center, Daicel Corporation, 1239 Shinzaike, Aboshi-ku, Himeji, Hyogo, 671-1283, Japan \\ ${ }^{2}$ Yoshimi Laboratories, Drug Safety Testing Center Co., Ltd., 25-1 Kuroiwa, Yoshimi-cho, Hiki-gun, Saitama, \\ 355-0166, Japan \\ ${ }^{3}$ Faculty of Engineering, Dept. of Materials Science and Engineering, Yokohama National University, \\ 79-5 Tokiwadai, Hodogaya, Yokohama, Kanagawa, 240-8501, Japan
}

(Received January 16, 2016; Accepted January 21, 2016)

\begin{abstract}
We previously reported the skin sensitizing potential of 41 different chemicals using a modified local lymph node assay with an elicitation phase (LLNA:DAE). Of these, the measured skin sensitizing potential for 39 was consistent with the reported results from a murine local lymph node assay (LLNA). Furthermore, the increase in the weight of the left ear lymph node per $1 \%$ test dose of each chemical (as measured using the LLNA:DAE method) seemed to reflect the class of skin sensitization potency as evaluated by LLNA. However, this relationship has never been quantitatively investigated. In the present study, we sought to determine the connection between the results of the LLNA:DAE method, in terms of lymph node weight, and the skin sensitization potency as indicated by the EC3 value. We evaluated the concentration of each chemical needed to increase the weight of the left ear lymph node by $2 \mathrm{mg}, 1 \mathrm{mg}, 0.5 \mathrm{mg}$, and $0.25 \mathrm{mg}$ during the elicitation phase using the LLNA:DAE method. These concentrations were then assessed in terms of how they directly compared to the LLNA EC3 value as well as how they matched the range of the EC3 value (under 1\%, 1-10\% and over 10\%). Finally, clear quantitative relationships between the EC values at $1 \mathrm{mg}$ and the $\mathrm{EC} 3$ values were observed using various statistical regression models. These results indicate that results obtained using the LLNA:DAE method can potentially be used to speculate the EC3 range found using LLNA method for these 29 kinds of skin sensitizers.
\end{abstract}

Key words: LLNA, LLNA:DAE, EC3

\section{INTRODUCTION}

Recently, many LLNA methods have adopted the Organization for Economic Co-operation and Development (OECD) test guidelines and have become the standard methods for testing skin sensitization. This mouse based testing model has several merits compared with traditional guinea pig methods, such as less animals being utilized, shorter testing periods and the ability to easily quantitate results (OECD 2002, 2010a, 2010b and 2010c; Haneke et al., 2001). However, one major issue with LLNAs is that they evaluate the skin sensitization potential of chemicals in the induction phase of the sensitization process, making it impossible to determine the cross-sensitization potential between chemicals. Some skin irritants are also known to give false positive results. For example, several skin irritants gave borderline positive results in the LLNA modified by Daicel based on ATP content (LLNA:DA) (ICCVAM, 2010). To resolve these issues, we previously developed the LLNA:DAE method (Yamashita et al., 2014, 2015a and 2015b). In this previous study, we reported the results of skin sensitization tests for 41 chemicals as well as the results of cross-sensitization tests between five chemical pairs. These results indicated that this method could in fact be used to evaluate the skin sensitization potential of chemicals as effectively as LLNA in

Correspondence: Kunihiko Yamashita (E-mail: ku_yamashita@jp.daicel.com) 
addition to allowing cross sensitization potentials among chemicals to be investigated.

Furthermore, we also previously implemented a skin sensitization scale that uses the results of the LLNA:DAE method to classify the potency of chemicals as extreme or strong, moderate, weak or non-skin sensitizer. In the current literature, the skin sensitization potency of chemicals is most often compared using the estimated concentration needed to produce a stimulation index of three (EC3) when using a LLNA. However, the relationship between the LLNA:DAE results and the EC3 value have never been investigated. Thus, in order to directly determine if the LLNA:DAE method is a more valuable tool for evaluating skin sensitization, we sought to quantitatively define the relationship between the elicitation response of the LLNA:DAE method and the EC3 values.

\section{Data analysis}

The LLNA:DAE lymph node weights used in this study as well as the 29 different chemicals used and EC3 values (\%) were all obtained from analyses conducted in our previous reports and another previous report, respectively (Yamashita et al., 2014, 2015a and 2015b; Nukada et al., 2013). In order to quantitatively analyze the relationship between the lymph node weight obtained using the LLNA:DAE method and EC3 values from the LLNA method, we first calculated the concentrations of each chemical needed in order to increase the weight of the lymph node in the left ear up to several defined levels when using the LLNA:DAE method. This increase in the weight of the left ear lymph node was obtained by subtracting that of control group mice from that of the test group. The concentrations of chemical which were needed to induce increases of $0.25 \mathrm{mg}, 0.5 \mathrm{mg}, 1 \mathrm{mg}$ and $2 \mathrm{mg}$ in lymph node weight were then compared to the EC3 values for that chemical. The calculated concentrations are expressed as "EC "\#" mg (\%)" in this study. For example, EC1mg means that this concentration, given as a percentage, would be expected to increase the weight of the lymph node in the left ear by $1 \mathrm{mg}$ during the elicitation phase for that chemical. Next, we divided skin sensitizing potency into three ranges referred to a previous report (Loveless et al., 2010): EC3 values below 1\%, EC3 values from $1 \%$ to $10 \%$, and EC3 values over $10 \%$. Each EC\#mg was then compared to these ranges to determine the most suitable way to estimate the EC3 range using only the results of the LLNA:DAE method.

Furthermore, to determine the relationship between the EC\#mg and EC3 values, three types of regression models were adopted, and the coefficients of determination were calculated to identify the statistical relationship between the two values. This analysis was performed using the statistical processing program mounted to Microsoft Excel 2010 (Microsoft, USA). For the exponential regression model and power exponential regression model, data were logarithm converted first and the regression equation was calculated.

\section{RESULTS}

The chemical name, lymph node weight, LLNA EC3 values and the calculated EC2mg, EC1mg, EC0.5mg and EC $0.25 \mathrm{mg}$ are listed in Table 1. Notably, when the skin sensitizing potency was classified into three ranges based on EC3 value (under 1\%, 1-10\% and over 10\%), the 29 chemicals were divided up as follows: 9 under 1\%, 7 between $1-10 \%$ and 13 over $10 \%$, when looking at their EC3 value alone. In our analysis, the calculated EC2mg, EC1mg, EC0.5mg and EC0.25mg values matched the LLNA EC3 value range for 16, 20, 18 and 18 of the 29 chemicals, respectively. Thus, it would appear that the EC1mg value may provide the best estimate when comparing the LLNA:DAE values to EC3 values in terms of the EC3 classification range. Furthermore, the number of chemicals found in the same under $1 \%, 1-10 \%$, and over $10 \%$ range as their EC3 value for the EC2mg (3, 0 and 13, respectively), EC1mg (5, 2 and 13), EC0.5mg (6, 4 and 8$)$, and EC0.25mg (9, 7 and 2) indicate that while the calculated EC1mg value may be the best overall estimator of EC3 value, EC0.25mg may be the best estimator when the potency concentration is suspected to be less than $10 \%$.

Using linear regression, we obtained formulas for the EC2mg $(y=0.4352 x-2.6703), E C 1 m g(y=0.8703 x-$ 2.6703), EC0.5mg $(\mathrm{y}=1.7406 \mathrm{x}-2.6703)$ and EC0.25mg $(y=3.4812 x-2.6703)$. The coefficient of determination for this linear regression model was 0.522 . Notably, the slope of the EC1mg formula was the closest to 1 . When the exponential regression model and the power exponential regression model were adopted, the coefficients of determination were 0.6276 and 0.87 , respectively. Thus, the power exponential model appears to give the highest coefficient of determination. The regression curve created using the power exponential model for the EC1mg compared with the EC3 values is shown in Fig. 1. These results indicate that there is a strong quantitative relationship between the calculated EC1mg and the EC3 values when the power exponential model is used. 
Quantitative analysis of the LLNA:DAE method results

Table 1. The LNW obtained by LLNA:DAE method, EC\#mg\% and EC3 values.

\begin{tabular}{|c|c|c|c|c|c|c|c|c|}
\hline Chemical name & $\mathrm{TC}(\%)$ & $\begin{array}{c}\text { LNW of LE } \\
\text { of TG }\end{array}$ & $\begin{array}{c}\text { LNW of LE } \\
\text { of CG }\end{array}$ & $\begin{array}{c}\mathrm{EC} 2 \mathrm{mg} \\
\%\end{array}$ & $\begin{array}{c}\text { EC1mg } \\
\%\end{array}$ & $\begin{array}{c}\mathrm{EC} 0.5 \mathrm{mg} \\
\%\end{array}$ & $\begin{array}{c}\mathrm{EC} 0.25 \mathrm{mg} \\
\%\end{array}$ & $\begin{array}{r}\text { LLNA } \\
(\mathrm{EC} 3) \%\end{array}$ \\
\hline 5-Chloro-2-methyl-4-isothiazolin-3-one & 0.05 & $2.7^{(a)}$ & $2.0^{\text {(a) }}$ & 0.14 & 0.07 & 0.04 & 0.02 & $0.005^{(\mathrm{c})}$ \\
\hline 2,4-Dinitrofluorobenzene & 0.25 & 7.3 (a) & $4.2^{\text {(a) }}$ & 0.16 & 0.08 & 0.04 & 0.02 & $0.03^{(\mathrm{c})}$ \\
\hline 2,4-Dinitrochlorobenzene & 0.25 & $5.1^{\text {(a) }}$ & $2.7^{\text {(a) }}$ & 0.21 & 0.10 & 0.05 & 0.03 & $0.05^{\text {(c) }}$ \\
\hline p-Benzoquinone & 1.00 & $5.3^{\text {(a) }}$ & $3.3^{(a)}$ & 1.00 & 0.50 & 0.25 & 0.13 & 0.0099 (c) \\
\hline Glutaraldehyde & 1.56 & 4.1 (a) & 2.4 (a) & 1.84 & 0.92 & 0.46 & 0.23 & $0.1^{(\mathrm{c})}$ \\
\hline Hydroquinone & 1.00 & $3.3^{\text {(a) }}$ & $2.3^{\text {(a) }}$ & 2.00 & 1.00 & 0.50 & 0.25 & $0.11^{(\mathrm{c})}$ \\
\hline p-Phenylenediamine & 2.50 & $3.3^{\text {(a) }}$ & $2.4^{(a)}$ & 5.56 & 2.78 & 1.39 & 0.69 & $0.16^{(\mathrm{c})}$ \\
\hline Propyl gallate & 6.25 & $3.9^{\text {(a) }}$ & $2.0^{\text {(a) }}$ & 6.58 & 3.29 & 1.64 & 0.82 & $0.32^{\text {(c) }}$ \\
\hline Cobalt chloride & 5.00 & $5.2^{\text {(a) }}$ & 2.7 (a) & 4.00 & 2.00 & 1.00 & 0.50 & $0.6^{(\mathrm{c})}$ \\
\hline Isoeugenol & 10 & $3.7^{\text {(a) }}$ & $2.5^{\text {(a) }}$ & 16.67 & 8.33 & 4.17 & 2.08 & $1.2^{(\mathrm{c})}$ \\
\hline 2-Mercaptobenzothiazole & 25 & $4.5^{\text {(a) }}$ & $2.8^{(\mathrm{a})}$ & 29.41 & 14.71 & 7.35 & 3.66 & $1.7^{\text {(c) }}$ \\
\hline Cinnamic aldehyde & 12.5 & $4.6^{(\mathrm{a})}$ & $2.2^{\text {(a) }}$ & 10.42 & 5.21 & 2.60 & 1.30 & $3.0^{(\mathrm{c})}$ \\
\hline Nichel sulphate & 25 & $3.6^{\text {(a) }}$ & $2.3^{\text {(a) }}$ & 38.46 & 19.23 & 9.62 & 4.81 & $4.80^{(\mathrm{c})}$ \\
\hline Maleic acid & 50 & $5.4^{(\mathrm{b})}$ & $3.4^{(b)}$ & 50.00 & 25.00 & 12.5 & 6.25 & $4.10^{\text {(d) }}$ \\
\hline Resorcinol & 25 & $3.2^{\text {(a) }}$ & $2.2^{\text {(a) }}$ & 50.00 & 25.00 & 12.50 & 6.25 & $5.70^{(\mathrm{c})}$ \\
\hline Linolenic acid & 25 & $3.4^{\text {(b) }}$ & $2.6^{(b)}$ & 62.50 & 31.25 & 15.63 & 7.81 & $9.80^{\text {(d) }}$ \\
\hline Oleic acid & 10 & $2.8^{(\mathrm{b})}$ & $2.1^{(b)}$ & 28.57 & 14.29 & 7.14 & 3.57 & $10.10^{\text {(d) }}$ \\
\hline Hexylcinnamic aldehyde & 25 & $4.0^{\text {(a) }}$ & $2.9^{\text {(a) }}$ & 45.45 & 22.73 & 11.36 & 5.68 & $11.00^{(\mathrm{c})}$ \\
\hline Eugenol & 50 & $3.8^{\text {(a) }}$ & $2.0^{(\mathrm{a})}$ & 55.56 & 27.78 & 13.89 & 6.94 & 13.00 (c) \\
\hline Citral & 25 & $5.1^{\text {(a) }}$ & $2.7^{\text {(a) }}$ & 20.83 & 10.42 & 5.21 & 2.60 & $13^{(\mathrm{c})}$ \\
\hline Linoleic acid & 25 & $3.4^{(b)}$ & $2.4^{(\mathrm{b})}$ & 50.00 & 25.00 & 12.50 & 6.25 & $14^{\text {(d) }}$ \\
\hline Abietic acid & 25 & $4.4^{(\mathrm{a})}$ & $2.4^{\text {(a) }}$ & 25.00 & 12.50 & 6.25 & 3.13 & $15^{\text {(c) }}$ \\
\hline Phenyl benzoate & 10 & $3.0^{\text {(a) }}$ & $2.1^{\text {(a) }}$ & 22.22 & 11.11 & 5.56 & 2.78 & 17 (c) \\
\hline Undecylenic acid & 25 & $3.1^{(b)}$ & $2.4^{(b)}$ & 71.43 & 35.71 & 17.86 & 8.93 & $19.4^{(\mathrm{d})}$ \\
\hline Cinnnamic alcohol & 25 & 3.5 (a) & $1.9^{(a)}$ & 31.25 & 15.63 & 7.81 & 3.91 & $21^{(\mathrm{c})}$ \\
\hline Imidazolidinyl urea & 40 & $3.3^{\text {(a) }}$ & $2.2^{\text {(a) }}$ & 72.73 & 36.36 & 18.18 & 9.09 & 24 (c) \\
\hline Ethylene glycol dimethacrylate & 40 & $3.1^{\text {(a) }}$ & $2.3^{\text {(a) }}$ & 100.00 & 50.00 & 25.00 & 12.50 & 28 (c) \\
\hline Hydroxycitronellal & 50 & 4.1 (a) & $2.5^{(a)}$ & 62.50 & 31.25 & 15.63 & 7.81 & 33 (c) \\
\hline Methyl methacrylate & 100 & $4.4^{(a)}$ & 2.3 (a) & 95.24 & 47.62 & 23.81 & 11.90 & $90^{\text {(c) }}$ \\
\hline
\end{tabular}

Abbreviations: TC (\%): Test concentrations; LNW of LE of TG: Left ear lymph node weight for the test group; LNW of LE of CG: Left ear lymph node weight for the control group. The values of lymph node weight and test concentrations were taken from (a) Yamashita et al. (2015a) and (b)Yamashita et al. (2015b). The EC3 values were taken from (c)Nukada et al. (2013) and (d)Yamashita et al. (2015b). Bold EC\#mg values indicate that it is in the same range (under 1\%, 1-10\% and over 10\%) as the EC3 value.

\section{DISCUSSION}

We previously reported the skin sensitization potential of 41 chemicals using the LLNA:DAE method. In this prior report, the skin sensitization potential of 39 out of the 41 chemicals was consists with the results of the LLNA method (Yamashita et al., 2014, 2015a and 2015b). These results indicate that both methods have the same potential to find out the skin sensitization potential for various chemicals. It is important to note that the underlying principles of the LLNA:DAE and LLNA methods are clearly different, with the raw data from each represent- ing a different biological response. However, a relationship between the elicitation response of LLNA:DAE and skin sensitization potency classification from the LLNA does exist. In the present study, we further investigated this relationship between the results of the LLNA:DAE and LLNA techniques using quantitative methodology.

To begin, we initially calculated the concentrations of each chemical that were necessary to induce a $2 \mathrm{mg}$, $1 \mathrm{mg}, 0.5 \mathrm{mg}$ and $0.25 \mathrm{mg}$ increase in left ear lymph node weight using the LLNA:DAE method. When the LLNA skin sensitization EC3 potency was divided into three classes (under 1\%,1-10\% and over 10\%), the EC1mg 

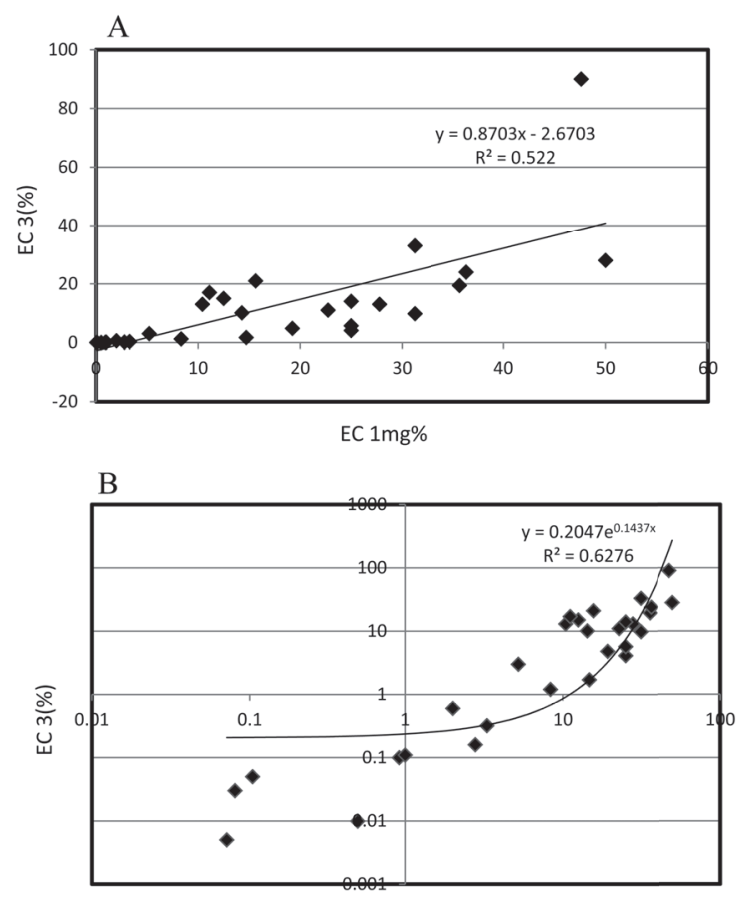

EC $1 \mathrm{mg} \%$

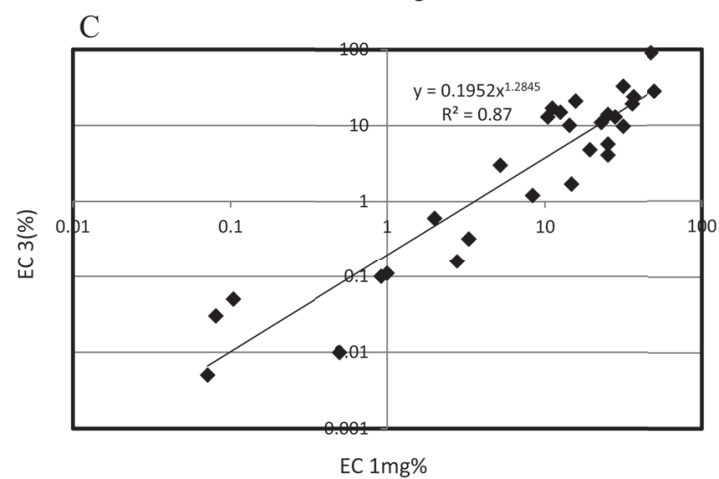

Fig. 1. Regression curves calculated comparing EC1mg and EC3 values of all 29 chemicals investigated in this study using basic linear regression (A), exponential regression $(\mathrm{B})$ and power exponential regression $(\mathrm{C})$. The formula and $\mathrm{R}^{2}$ values (coefficient of determination) are provided. The $\mathrm{X}$ axis indicates the EC1mg (\%) values, while the $\mathrm{Y}$ axis indicates the EC3 values.

value appeared to agree the most with the EC3 classification, with 20 out of 29 of the chemicals being in the same range bracket with both methods. Thus, it may be likely that the LLNA:DAE EC1mg values could actually predict the LLNA EC3 values for 20 of the 29 chemicals investigated. However, it is important to note that 23 out of 29 EC1mg values were higher than the actual EC3 values, even though they were still within the same EC3 range.
We believe this may reflect the differences in the sensitization phase the LLNA:DAE (elicitation phase) and LLNA (induction phase) methods analyze. Our data are corroborated by the results of the OECD TG429 report that evaluated an alternative method of LLNA, which set the same acceptable EC3 range and used values from $0.5 \times \mathrm{EC} 3$ to $2 \times \mathrm{EC} 3(\mathrm{OECD}, 2010 \mathrm{~b})$. The number of EC2mg, EC1mg, EC0.5mg and EC0.25mg values that were in an acceptable range with the EC3 value for the chemicals investigated 4, 12, 12 and 9, respectively. Taken together with our results, it appears that EC1mg values obtained using LLNA:DAE methods could be used to estimate the range of the EC3 values, but these values are only an estimate as it is difficult to estimate the exact EC3 values from the results of the alternative methods directly.

Next, we investigated the relationship between the elicitation response measured with the LLNA:DAE method and the induction response indicated by the EC3 values using statistical analysis tools. The tritium thymidine incorporated by the LLNA method increases exponentially following application of some chemicals application, and T-cell proliferation is affected by various biological factors, including cytokines (van Och et al., 2000; Morris et al., 2009). Based on this consideration, we analyzed our EC1mg and EC3 data with a linier regression model, exponential regression model, and power exponential regression model and compared their fit to the data. The coefficients of determination were 0.522 , 0.6276 and 0.87 , respectively. Thus, it would appear that the power regression model has the best fit to our data. The underlying reasons why this power regression model gives the highest coefficient of determination value is unclear, but we believe it may reflect the exponential lymph node cell increase caused by the chemical. In fact, this statistical test is known to indicate strong correlations between the two parameters (Guilford, 1956). This value could therefore also indicate that there is a strong correlation between the EC1mg and EC3 values, supporting our previous analysis and highlighting the possible use of the LLNA:DAE EC1mg to provide a reasonable estimate of the EC3 value.

In conclusion, although the predictive performance of the LLNA:DAE method for skin sensitization potential of chemicals was previously reported, we have for the first time provided quantitative evidence that a relationship between the lymph node weight obtained with the LLNA:DAE method and the LLNA EC3 values exists. The principles and protocols for the two methods are notably different; however, it appears that the endpoint of these analyses was similar for the chemicals evaluated in this study in that lymph node proliferation was in fact 
Quantitative analysis of the LLNA:DAE method results

induced by chemical application. Thus, it appears that the observed quantitative relationship between the results of both methods is meaningful and that the EC3 values could be estimated from the results of the LLNA:DAE method. Further analysis to determine the most accurate way to predict EC3 values from the results of LLNA:DAE method is needed, emphasizing additional skin sensitizing chemicals.

Conflict of interest---- The authors declare that there is no conflict of interest.

\section{REFERENCES}

Guilford, J.P. (1956): Fundamental statistics in psychology and education. ( ${ }^{\text {rd }}$ ed.). New York: McGraw-Hill.

Haneke, K.E., Tice, R.R., Carson, B.L., Margolin, B.H. and Stokes, W.S. (2001): ICCVAM evaluation of the murine local lymph node assay. Data analyses completed by the National Toxicology Program Interagency Center for the Evaluation of Alternative Toxicological Methods. Regul. Toxicol. Pharmacol., 34, 274286.

ICCVAM (2010): ICCVAM test method evaluation report on the murine local lymph node assay: DA, a nonradioactive alternative test method to assess the allergic contact dermatitis potential of chemicals and products. NIH Publication No. 10-7551. Research Triangle Park, NC: National Institute of Environmental Health Sciences. New York: McGraw-Hill.

Loveless, S.E., Api, A.-M., Crevel, R.W.R., Debruyne, E., Gamer, A., Jowsey, I.R., Kern, P., Kimber, I., Lea, L., Lloyd, P., Mehmood, Z., Steiling, W., Veenstra, G., Woolhiser, M. and Hennes,C. (2010): Potency values from the local lymph node assay; Application to classification, labelling and risk assessment. Regul. Toxicol. Pharmacol., 56, 54-65.

Morris, S.C., Heidorn, S.M., Herbert, De'B.R., Perkins, C., Hildeman, D.A., Khodoun, M.V. and Finkelman, F.D. (2009): Endogenously produced IL-4 nonredundantly stimulates $\mathrm{CO}^{+} \mathrm{T}$ cell proliferation. J. Immunol., 182, 1429-1438.
Nukada, Y., Miyazawa, M., Saitoh, K., Sakaguchi, H. and Nishiyama, H. (2013): Data integration of non-animal tests for the development of a test battery to predict the sensitozing potential and potency of chemicals. Toxicol. Vitr., 27, 609-618.

OECD (2002): OECD guideline for the testing of chemicals, test no. 429: Skin sensitization: local lymph node assay, adopted July 2002. Paris, France: Organisation for Economic Cooperation and Development.

OECD (2010a): OECD guideline for the testing of chemicals, test no. 442A: Skin sensitization: local lymph node assay: DA, adopted July 2010. Paris, France: Organisation for Economic Cooperation and Development.

OECD (2010b): OECD guideline for the testing of chemicals, test no. 429.: Skin sensitization: local lymph node assay, adopted July 2010. Paris, France: Organisation for Economic Cooperation and Development.

OECD (2010c): OECD guideline for the Testing of Chemicals, Test No.429.: Skin sensitization: Local Lymph Node Assay BrdUELISA, adopted July 2010. Paris, France: Organization for Economic Cooperation and Development.

Van Och, F.M.M., Slob, W., Jong, W.H., Vandebriel, R.J. and Loveren, H. (2000): A quantitative method for assessing the sensitizing potency of low molecular weight chemicals using a local lymph node assay: employment of a regression method that includes determination of the uncertainty margins. Toxicology, 146, 49-59.

Yamashita, K., Shinoda, S., Hagiwara, S. and Itagaki, H. (2014): Development of LLNA:DAE: a new local lymph node assay that includes the elicitation phase, discriminates borderline-positive chemicals, and is useful for cross-sensitization testing. J. Toxicol. Sci., 39, 147-161.

Yamashita, K., Shinoda, S., Hagiwara, S. and Itagaki, H. (2015a): Further development of LLNA:DAE method as stand-alone skin-sensitization testing method and applied for evaluation of relative skin-sensitizing potency between chemicals. J. Toxicol. Sci., 40, 137-150.

Yamashita, K., Shinoda, S., Hagiwara, S., Miyazaki, H. and Itagaki, H. (2015b): Unsaturated fatty acids show clear elicitation responses in a modified local lymph node assay with an elicitation phase, and test positive in the direct peptide reactivity assay. J. Toxicol. Sci., 40, 843-853. 